\title{
Primeros registros documentados de la mojarra Cheirodon ibicuhiensis Eigenmann, 1915 (Characiformes, Cheirodontinae) para la provincia de Córdoba, Argentina
}

\section{First documented records of Cheirodon ibicuhiensis Eigenmann, 1915 (Characiformes, Cheirodontinae) for Córdoba province, Argentina}

\author{
Natalia G. Rivetti ${ }^{1,2}$, Andrea Hued ${ }^{1,2}$, Alejo Bonifacio ${ }^{3}$, María laura Ballesteros ${ }^{1,2}$, María A. \\ BISTONI, ${ }^{1,2}$. \\ ${ }^{1}$ Consejo Nacional de Investigaciones Científicas y Técnicas (CONICET), Instituto de Diversidad y Ecología \\ Animal (IDEA). Córdoba, Argentina. \\ ${ }^{2}$ Universidad Nacional de Córdoba, Facultad de Ciencias Exactas, Físicas y Naturales. Cátedra de Diversidad \\ Biológica IV. Córdoba, Argentina. \\ ${ }^{3}$ Universidad Nacional de Córdoba, Facultad de Ciencias Exactas, Físicas y Naturales. Cátedra de Morfología \\ Animal. Córdoba, Argentina.
}

E-mail: nattyrivetti@gmail.com

RESUMEN. En este trabajo, se reporta por primera vez la presencia de la mojarra Cheirodon ibicuhiensis para la cuenca del río Primero (Suquía) en la provincia de Córdoba, Argentina. Esta especie había sido citada en Argentina para las secciones media e inferior de los ríos Uruguay y Paraná. Estos registros de C. ibicuhiensis en la provincia de Córdoba amplían su distribución a otras cuencas no pertenecientes a la cuenca del Plata en Argentina. Los ejemplares fueron observados en la región de las Sierras Chicas de Córdoba, en los últimos diez años, en cuatro ríos serranos: Chorrillos, Toro Muerto, Yuspe y Suquía. Se exponen las medidas morfométricas de nueve ejemplares de esta mojarra y los parámetros ambientales del hábitat donde fue registrada. Esta especie habría sido introducida a los ríos serranos de la Córdoba con el fin de mantener ejemplares vivos para ser utilizados como carnada. Siempre se la ha observado formando cardúmenes con otras mojarras autóctonas de la provincia.

Palabras clave: Cheirodontidae, distribución, ictiofauna de Córdoba, Sierras Chicas.

\begin{abstract}
In this work, the presence of Cheirodon ibicuhiensis is reported for the first time in the Primero River basin (Suquía) in the province of Córdoba, Argentina. This species had been previously reported in Argentina for the median and lower sections of the Uruguay and Paraná rivers. However, these new records of $C$. ibicuhiensis for the province of Córdoba extend its distribution to other basins outside of the east region of Argentina. The species have been observed in the Sierras Chicas region in the last decade, in four rivers: Chorrillos, Toro Muerto, Yuspe and Suquía. Morphometric measurements of nine specimens of this species and environmental parameters of the habitat where it was collected are also provided. Probably, C. ibicuhiensis was introduced into this river in order to have specimens for live-bait for fishing. It has always been observed forming shoals with other native mojarras of the province.
\end{abstract}

Keywords: Cheirodontidae, distribution, ichthyofauna of Córdoba, Sierras Chicas. 


\section{INTRODUCCIÓN}

En Argentina, la subfamilia Cheirodontinae comprende actualmente cinco géneros: Cheirodon, Heterocheirodon, Macropsobrycon, Odontostilbe y Serrapinnus. Dentro del género Cheirodon, dos especies de mojarras son reconocidas: Cheirodon interruptus (Jenyns, 1842) y Cheirodon ibicuhiensis Eigenmann, 1915. De estas, C. interruptus es la que se encuentra más ampliamente distribuida en las cuencas de la Argentina (Mantinian et al., 2008).

Cheirodon ibicuhiensis fue reconocida como especie por Malabarba (1989). Posteriormente, Mantinian et al. (2008) realizaron una descripción más completa que incluyó aspectos reproductivos y ecológicos. La distribución global de la especie abarca diversas cuencas de Argentina, Brasil y Uruguay. Particularmente en la Argentina, diversos autores reportaron su presencia en las secciones media e inferior de los ríos Uruguay y Paraná, así como en algunos de sus afluentes (Malabarba, 2003; Mantinian et al., 2008; Minotti, 2011; Miquelarena et al., 2008; Scarabotti et al., 2011).

Esta mojarra se distingue de las demás especies del género por su cuerpo relativamente alto (28,2-39,8\% de la longitud estándar) y comprimido, el elevado número de radios ramificados en la aleta anal (19 a 23) y bajo número de radios caudales procurrentes inferiores (17 a 22). En la aleta anal de los machos adultos se distinguen de dos a tres pares de pequeñas espinas por segmento de lepidotriquia (Mantinian et al., 2008).

En la provincia de Córdoba, la única especie del género Cheirodon registrada hasta el momento había sido $C$. interruptus, citada para todas las principales cuencas de la provincia (Haro y Bistoni, 2007; Menni, 2004). El objetivo de esta comunicación es reportar por primera vez la presencia de C. ibicuhiensis para la cuenca del río Primero (Suquía) en la provincia de Córdoba.

\section{MATERIALES Y MÉTODOS}

La observación y recolección de ejemplares se realizó en el marco de relevamientos de fauna íctica y monitoreos de calidad de agua llevados a cabo en la región de las Sierras Chicas de Córdoba en los últimos diez años. Los muestreos de peces se realizaron con un equipo de pesca eléctrica estándar (SmithRootLR-20B) y redes de mano para la captura en lugares de difícil acceso. Los ejemplares de $C$. ibicuhiensis fueron recolectados e identificados en laboratorio a través del uso de claves dicotómicas (Miquelarena et al., 2008). Para cada sitio de recolección se midieron in situ los siguientes parámetros físico y químicos del agua: temperatura, $\mathrm{pH}$, conductividad, turbidez, oxígeno disuelto y sólidos disueltos totales con una sonda multiparamétrica Horiba U-52. Además, se caracterizó la estructura del hábitat a través de las siguientes variables: profundidad de los cursos de agua (promedio entre zona de costa y zona media del cauce), área del cauce muestreada (calculada en Google Earth) y estimación visual de vegetación acuática presente en el área (porcentaje de ocupación). Los ejemplares de C. ibicuhiensis fueron conservados en formol al 10\% y trasladados a laboratorio. El material recolectado se encuentra depositado en la cátedra de Diversidad Biológica IV, Facultad de Ciencias Exactas, Físicas y Naturales en la Universidad Nacional de Córdoba. En estos individuos se tomaron 32 medidas morfológicas con una precisión de $0,001 \mathrm{~mm}$. Las mediciones se realizaron a partir de los ejemplares fotografiados, con el software Image J v. 1.53i (Rasband, 2012).

\section{RESULTADOS}

Cheirodon ibicuhiensis fue registrada en la cuenca del río Primero (Suquía) en las Sierras Chicas de Córdoba en los siguientes ríos: río Los Chorrillos, en las localidades de Santa Cruz del

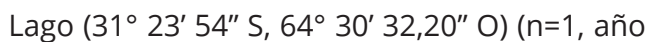
2019), de Villa Carlos Paz ( $31^{\circ} 23^{\prime} 43,50^{\prime \prime}$ S, $64^{\circ}$ $32^{\prime} 25,20^{\prime \prime}$ O) (n=9, año 2019) y de Estancia Vieja (312 23'35.46" S, 64 31' 16.23" O) (ejemplares no contabilizados, año 2021); río Toro Muerto, localidad de Villa Flor Serrana (31 $23^{\circ} 20,00^{\prime \prime}$ $\left.\mathrm{S}, 64^{\circ} 36^{\prime} 4,70^{\prime \prime} \mathrm{O}\right)$ ( $\mathrm{n}=2$, año 2019); río Yuspe, localidad de Cosquín (31 14' 25" S,64 31' 16" 
O) (n=1, año 2014); río Suquía, en las localidades de Casa Bamba ( $31^{\circ} 21^{\prime}$ 0,30' S, 64 23' 50,43" O) (ejemplares no contabilizados, año 2015)

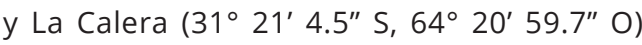
(ejemplares no contabilizados, año 2021). Los datos morfométricos y merísticos registrados para los nueve ejemplares capturados se exponen en la Tabla 1. En la Figura 1 se observa un ejemplar capturado en la localidad de La
Calera (río Suquía).

Los individuos fueron recolectados a altitudes comprendidas entre los 400 y 800 m s.n.m., en cursos lóticos serranos típicos de la región, con aguas cristalinas, escasa pendiente y a una profundidad menor a $2 \mathrm{~m}$. La especie fue encontrada fundamentalmente en cardúmenes próximos a las riberas de los cursos, en sustratos de tipo arenoso y grava, con presencia

Tabla 1. Datos morfométricos y merísticos de Cheirodon ibicuhiensis $(\mathrm{n}=9)$ colectados en la Provincia de Córdoba, Argentina (DE: desvío estándar).

Table 1. Morphometric data of Cheirodon ibicuhiensis ( $n=9)$ collected in the Province of Córdoba, Argentina (DE: standard deviation).

\begin{tabular}{|c|c|c|c|c|c|c|}
\hline \multirow{2}{*}{ Rasgos } & \multicolumn{6}{|c|}{ Ejemplares } \\
\hline & 1 & 2 & 3 & 4 & 5 & 6 \\
\hline Longitud total (mm) & 45,28 & 34,77 & 43,03 & 43,10 & 40,54 & 41,39 \\
\hline Longitud estándar (mm) & 36,56 & 28,80 & 33,76 & 35,64 & 33,17 & 33,58 \\
\hline № escamas longitudinales & 33 & $28-30 *$ & 33 & 36 & 35 & 33 \\
\hline № escamas perforadas & 7 & 8 & 8 & 9 & 6 & 9 \\
\hline Altura de la línea media del cuerpo & 6,69 & 5,41 & 5,76 & 6,454 & 6,25 & 6,341 \\
\hline Altura máxima del cuerpo (mm) & 12,55 & 9,83 & 11,73 & 13,57 & 10,43 & 11,25 \\
\hline Altura del cuerpo / Long. estándar (\%) & 34,33 & 34,13 & 34,75 & 38,08 & 31,44 & 33,50 \\
\hline Ancho máximo del cuerpo (mm) & 4,66 & 3,83 & 4,45 & 4,41 & 5,08 & 4,17 \\
\hline Altura de la cabeza(mm) & 5,91 & 4,93 & 5,90 & 5,65 & 6,13 & 6,17 \\
\hline Ancho de la cabeza (mm) & 4,397 & 3,94 & 4,05 & 3,82 & 4,41 & 3,79 \\
\hline Longitud de la cabeza (mm) & 7,81 & 7,54 & 8,40 & 8,64 & 8,03 & 8,15 \\
\hline Longitud del hocico (mm) & 1,41 & 1,34 & 1,70 & 1,52 & 1,56 & 1,39 \\
\hline Altura del ojo (mm) & 3,66 & 2,89 & 3,13 & 3,37 & 3,38 & 3,69 \\
\hline Diámetro del ojo (mm) & 3,17 & 2,89 & 2,85 & 3,04 & 3,03 & 3,10 \\
\hline Ancho del pedúnculo caudal (mm) & 1,28 & 0,95 & 1,096 & 1,25 & 1,27 & 1,24 \\
\hline Longitud del pedúnculo caudal (mm) & 6,07 & 5,36 & 5,64 & 5,75 & 5,65 & 4,33 \\
\hline Altura del pedúnculo caudal (mm) & 5,14 & 3,796 & 4,51 & 5,04 & 5 & 4,81 \\
\hline Altura aleta anal (mm) & 3,80 & 3,298 & 3,51 & 3,75 & 4,72 & 5,01 \\
\hline Longitud aleta anal (mm) & 9,82 & 7,10 & 7,52 & 8,84 & 7,24 & 6,79 \\
\hline Long. aleta anal / Long. Estándar (\%) & 26,86 & 24,65 & 22,28 & 24,80 & 21,83 & 20,22 \\
\hline № radios ramificados aleta anal & 21 & 20 & 22 & 20 & $17-18$ & 20 \\
\hline Altura aleta caudal(mm) & 7,54 & 7,43 & 8,54 & 7,70 & 10,92 & 10,66 \\
\hline Longitud aleta caudal (mm) & 9,23 & 8,38 & 7,99 & 9,64 & 9,298 & 9,41 \\
\hline № radios caudales procurrentes & $20-21$ & $20-21$ & $21-22$ & 19 & $16-17$ & 20 \\
\hline Altura aleta dorsal (mm) & 3,04 & 2,96 & 2,85 & 3,03 & 3,61 & 3,80 \\
\hline Longitud aleta dorsal (mm) & 9,83 & 8,41 & 7,59 & 9,42 & 9,86 & 10,15 \\
\hline Altura aleta pectoral (mm) & 1,50 & 1,22 & 1,39 & 1,76 & 1,41 & 1,34 \\
\hline Longitud aleta pectoral (mm) & 8,67 & 6,32 & 6,10 & 7,395 & 7,21 & 6,84 \\
\hline Altura aleta pélvica (mm) & 1,52 & 1,19 & 1,17 & 1,64 & 1,197 & 1,37 \\
\hline Longitud aleta pélvica (mm) & 6,65 & 5,37 & 7,49 & 6,32 & 7,35 & 7,17 \\
\hline
\end{tabular}




\begin{tabular}{|c|c|c|c|c|c|}
\hline \multirow{3}{*}{ Rasgos } & \multicolumn{3}{|c|}{ Ejemplares } & \multirow{3}{*}{ Media } & \multirow{3}{*}{ DE } \\
\hline & & & & & \\
\hline & 7 & 8 & 9 & & \\
\hline Longitud total (mm) & 40,07 & 41,10 & 37,10 & 40,71 & 3,19 \\
\hline Longitud estándar (mm) & 33,88 & 35,38 & 32,61 & 33,71 & 2,24 \\
\hline № escamas longitudinales & 34 & 33 & $28-30 *$ & 34 & 1 \\
\hline № escamas perforadas & 7 & 7 & 8 & 8 & 1 \\
\hline Altura de la línea media del cuerpo & 5,63 & 6,06 & 6,11 & 6,08 & 0,41 \\
\hline Altura máxima del cuerpo (mm) & 11,87 & 12,36 & 11,32 & 11,66 & 1,13 \\
\hline Altura del cuerpo / Long. estándar (\%) & 35,04 & 34,94 & 34,71 & 34,55 & 1,73 \\
\hline Ancho máximo del cuerpo (mm) & 3,95 & 4,85 & 4,11 & 4,39 & 0,42 \\
\hline Altura de la cabeza(mm) & 5,91 & 5,78 & 5,53 & 5,77 & 0,38 \\
\hline Ancho de la cabeza (mm) & 3,43 & 4,02 & 3,63 & 3,96 & 0,33 \\
\hline Longitud de la cabeza (mm) & 7,53 & 7,82 & 7,46 & 7,93 & 0,41 \\
\hline Longitud del hocico (mm) & 1,84 & 1,48 & 1,44 & 1,52 & 0,16 \\
\hline Altura del ojo (mm) & 3,17 & 3,099 & 3,09 & 3,27 & 0,27 \\
\hline Diámetro del ojo (mm) & 3,03 & 2,96 & 2,72 & 2,98 & 0,14 \\
\hline Ancho del pedúnculo caudal (mm) & 1,12 & 1,34 & 1,20 & 1,19 & 0,12 \\
\hline Longitud del pedúnculo caudal (mm) & 4,28 & 4,296 & 4,87 & 5,14 & 0,70 \\
\hline Altura del pedúnculo caudal (mm) & 4,65 & 4,94 & 4,95 & 4,76 & 0,41 \\
\hline Altura aleta anal (mm) & 4,44 & 4,296 & 3,94 & 4,09 & 0,57 \\
\hline Longitud aleta anal (mm) & 8,01 & 8,37 & 8,26 & 7,99 & 0,96 \\
\hline Long. aleta anal / Long. Estándar (\%) & 23,64 & 23,66 & 25,33 & 23,70 & 2,01 \\
\hline № radios ramificados aleta anal & 19 & 21 & $16-17$ & 20 & 1 \\
\hline Altura aleta caudal(mm) & 9,57 & 10,42 & 7,52 & 8,92 & 1,48 \\
\hline Longitud aleta caudal (mm) & 6,74 & 9,44 & 8,48 & 8,77 & 0,93 \\
\hline № radios caudales procurrentes & 19 & 22 & $20-21$ & 20 & 2 \\
\hline Altura aleta dorsal (mm) & 3,66 & 5,17 & 3,79 & 3,55 & 0,72 \\
\hline Longitud aleta dorsal (mm) & 9,14 & 10,49 & 9,02 & 9,33 & 0,91 \\
\hline Altura aleta pectoral (mm) & 1,48 & 1,69 & 1,496 & 1,48 & 0,17 \\
\hline Longitud aleta pectoral (mm) & 6,27 & 6,51 & 7,00 & 6,92 & 0,79 \\
\hline Altura aleta pélvica (mm) & 1,27 & 1,38 & 1,52 & 1,36 & 0,17 \\
\hline Longitud aleta pélvica (mm) & 5,64 & 7,53 & 6,67 & 6,69 & 0,79 \\
\hline
\end{tabular}

*El recuento no es preciso debido a que los ejemplares han perdido algunas escamas durante la manipulación y el proceso de conservación.

*The count is not accurate because the specimens have Ist some scales during handling and preservation process. 


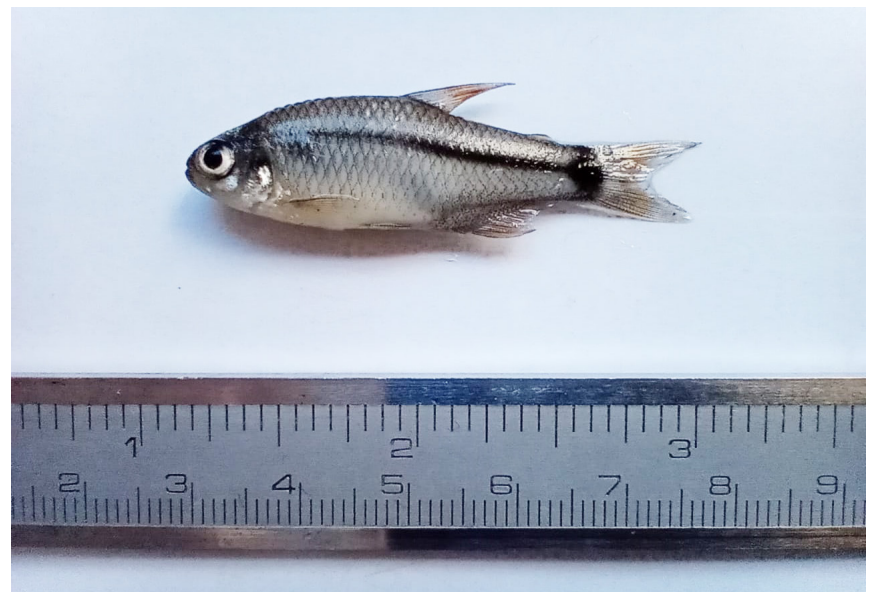

Figura 1. Ejemplar de Cheirodon ibicuhiensis capturado en el río Suquía en la localidad de La Calera (Córdoba). Figure1. Specimen of Cheirodon ibicuhiensis captured in the Suquía River, at the locality of La Calera (Córdoba).

de vegetación acuática (algas y macrófitas). En la Tabla 2 se resumen las variables físicas, químicas y de hábitat registradas para caracterizar los sitios donde se capturaron los ejemplares.

\section{DISCUSIÓN}

Los registros de C. ibicuhiensis en ríos serranos de la cuenca del río Primero son relevantes ya que constituyen las primeras evidencias documentadas que permiten confirmar la presencia de esta especie en la provincia de Córdoba. De esta manera, estos registros amplían la distribución de esta especie a otras cuencas no pertenecientes la cuenca del Plata en la Argentina. Según pescadores de la zona, esta mojarra habría sido introducida desde la región mesopotámica a los ríos serranos de la provincia de Córdoba con el fin de mantener los ejemplares vivos para ser utilizados como carnada.

Cheirodon ibicuhiensis comparte el hábitat con otras especies de mojarras formando cardúmenes mixtos. A modo de ejemplo, en los registros de la localidad de Santa Cruz del Lago, se capturaron nueve ejemplares de C. ibicuhiensis, 84 de C. interruptus y tres de Psalidodon sp., formando parte del mismo cardumen (obs. pers.).

Debe destacarse el ambiente contrastante

Tabla 2. Variables físico y químicas del agua y de hábitat de los sitios donde Cheirodon ibicuhiensis fue recolectada (DE: desvío estándar).

Table 2. Physical, chemical and habitat variables of the sampling sites where Cheirodon ibicuhiensis was collected (DE: standard deviation).

\begin{tabular}{llccc}
\hline & Parámetros & Rango & Media & DE \\
\hline Físicos y Químicos & Temperatura $(\mathrm{O} C)$ & $13,57-16,16$ & $\mathbf{1 3 , 5 4}$ & $\mathbf{1 , 3 1}$ \\
& $\mathrm{pH}$ & $6,00-9,49$ & $\mathbf{7 , 8 5}$ & $\mathbf{1 , 7 5}$ \\
& Oxígeno disuelto $(\mathrm{mg} / \mathrm{L})$ & $11,04-12,46$ & $\mathbf{1 1 , 8 3}$ & $\mathbf{0 , 4 9 9}$ \\
& Conductividad $(\mathrm{\mu S} / \mathrm{cm})$ & $87-318$ & $\mathbf{1 5 7}$ & $\mathbf{1 2 8}$ \\
& Turbidez $(\mathrm{NTU})$ & $0,00-7,33$ & $\mathbf{2 , 3 6}$ & $\mathbf{1 , 0 7}$ \\
& Sólidos Totales Disueltos $(\mathrm{g} / \mathrm{L})$ & $0,06-0,21$ & $\mathbf{0 , 1 0}$ & $\mathbf{0 , 0 8}$ \\
& Profundidad $(\mathrm{m})$ & $0,29-0,42$ & $\mathbf{0 , 3 5}$ & $\mathbf{0 , 0 6}$ \\
& Área de cauce $($ ha) & $0,24-0,28$ & $\mathbf{0 , 3 1}$ & $\mathbf{0 , 1 4}$ \\
& \% Vegetación acuática & $0,00-79,58$ & $\mathbf{1 7 , 9 7}$ & $\mathbf{1 7 , 3 1}$ \\
\hline
\end{tabular}


donde se ha capturado a la especie en la Provincia de Córdoba en comparación a la descripción realizada por Mantinian et al. (2008), quienes mencionan haberla capturado en lagos de llanura con fondo fangoso, agua turbia y baja concentración de oxígeno.

La presente nota pone de manifiesto la importancia de llevar a cabo periódicamente relevamientos de la fauna íctica en los cuerpos de agua continentales. De este modo, y de acuerdo con lo expuesto por los pescadores de la zona, se evidencian procesos, tales como la antropocoria, que afectan a la distribución original de la ictiofauna. A futuro, sería interesante relevar cuencas aledañas a las del presente estudio y determinar si la especie está presente también en ellas.

\section{REFERENCIAS}

Haro, J. G. y Bistoni, M. A. (2007). Peces de Córdoba. Córdoba: Editorial Universidad Nacional de Córdoba.

Malabarba, L. R. (1989). Histórico sistemático e lista comentada das espécies de peixes de água doce do sistema da Laguna dos Patos, Rio Grande do Sul, Brasil. Comunicações do Museu de Ciências e Tecnologia UBEA/PUCRS, Série Zoologia, 2(8), 107-179.

Malabarba, L. R. (2003). Subfamily Cheirodontinae (Characins, tetras). In: Reis, R.E., Kullander, S. \& Ferraris, C. J. (Eds.). Check list of the freshwater fishes of South and Central America (pp. 215-221). Porto Alegre: EDIPUCRS.

Mantinian, J., Miquelarena, A. \& Scarabotti, P. (2008). Redescription of Cheirodon ibicuiensis Eigenmann, 1915 (Characiformes: Cheirodontinae), with notes on its distribution in Argentina. Natura Neotropicalis, 1(39), 33-46. https://doi.org/10.14409/natura. v1i39.3867.

Menni, R. C., López, H. L., Casciotta, J. R. y Miquelarena, A. M. (1984). Ictiología de áreas serranas de Córdoba y San Luis (Argentina). Biología Acuática, 5, 2-63. Disponible en: http://naturalis.fcnym.unlp. edu.ar/id/000201

Minotti, P. (2011). Fauna íctica que habita las arroceras del noreste de Argentina. En: Blanco, D. E. y de la Balze, V. (Eds.) Conservación de los recursos acuáticos y la biodiversidad en arroceras del noreste de Argentina (pp. 41-61). Buenos Aires: Fundación para la Conservación y el Uso Sustentable de los Humedales. Disponible en: https://lac.wetlands.org/publicacion/ conservacion-de-los-recursos-acuaticos-yla-biodiversidad-en-arroceras-del-norestede-argentina-2/

Miquelarena, A. M., Mantinian, J. y López, H. (2008). Peces de la Mesopotamia Argentina (Characiformes: Characidae: Cheirodontinae). Temas de la Biodiversidad del Litoral fluvial argentino III. INSUGEO. Miscelánea, 17, 51-90. Disponible en: http://www.insugeo.org.ar/ scg/ver-articulo.php?id=371

Rasband, W. S. (2012). ImageJ. U.S. National Institutes of Health, Bethesda, Maryland, USA, 1; 1997-2012.

Scarabotti, P., López, J. \& Pouilly, M. (2011). Flood pulse and the dynamics of fish assemblage structure from neotropical floodplain lakes. Ecology of Freshwater Fish, 20,605 - 618. https://doi.org/10.1111/j.16000633.2011.00510.x 